\title{
Making biobank data and samples findable and accessible
}

David van Enckevort ${ }^{1}$, Erik van Iperen ${ }^{2}$, Barteld Klasens ${ }^{3}$, Gijs Kant ${ }^{3}$, Riza Nugraha ${ }^{3}$, Jolanda Strubel ${ }^{3}$, Janneke Schoots ${ }^{3}$, BIOS consortium, GO-NL consortium, Parelsnoer Institute, PALGA, MOLGENIS Developers, Jeroen Beliën ${ }^{4}$, Jan-Willem Boiten ${ }^{5}$,Morris Swertz ${ }^{1}$

${ }^{1}$ Department of Genetics, University Medical Center Groningen, Groningen, ${ }^{2}$ Durrer Center for Cardiovascular Research; ${ }^{3}$ The Hyve, Utrecht; ${ }^{4} \mathrm{VU}$ University Medical Center, Amsterdam; ${ }^{5}$ Lygature, Utrecht

\section{SOLUTION}

To improve the utilization of existing biobank collections and improve the access to material BBMRI-NL 2.0 has further developed the MOLGENIS Catalogue and the Podium request workflow. The catalogue makes biobank samples and data findable and accessible aiming to increase use of biobank resources.

Podium eases the data and sample request with a complete track and trace of the request workflow. This reduces the workload for both the researcher and the biobanks and facilitates fast recovery recalled material, proper provenance and audit trail.

- Researcher finds relevant
biobanks in the Catalogue

All software is available as open source: MOLGENIS: https://github.com/molgenis/molgenis Podium: https://github.com/thehyve/podium

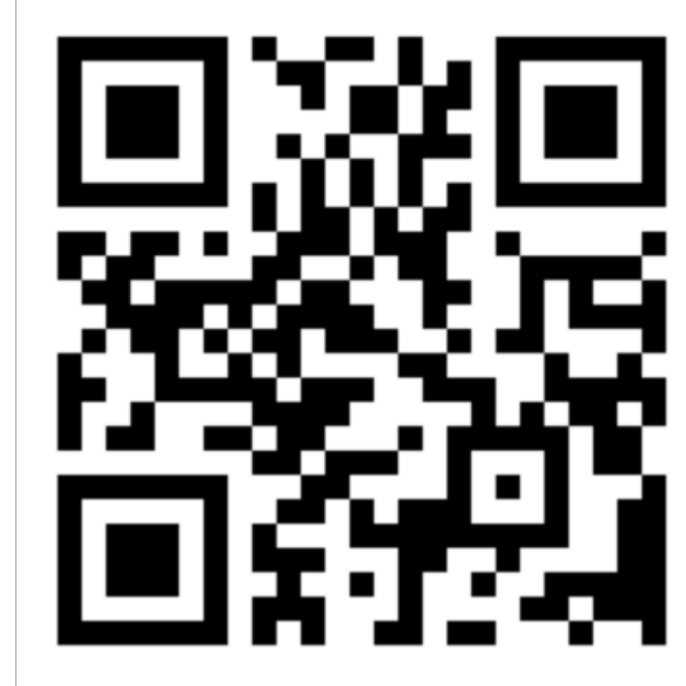
molgenis catalogue

\section{CATALOGUE}

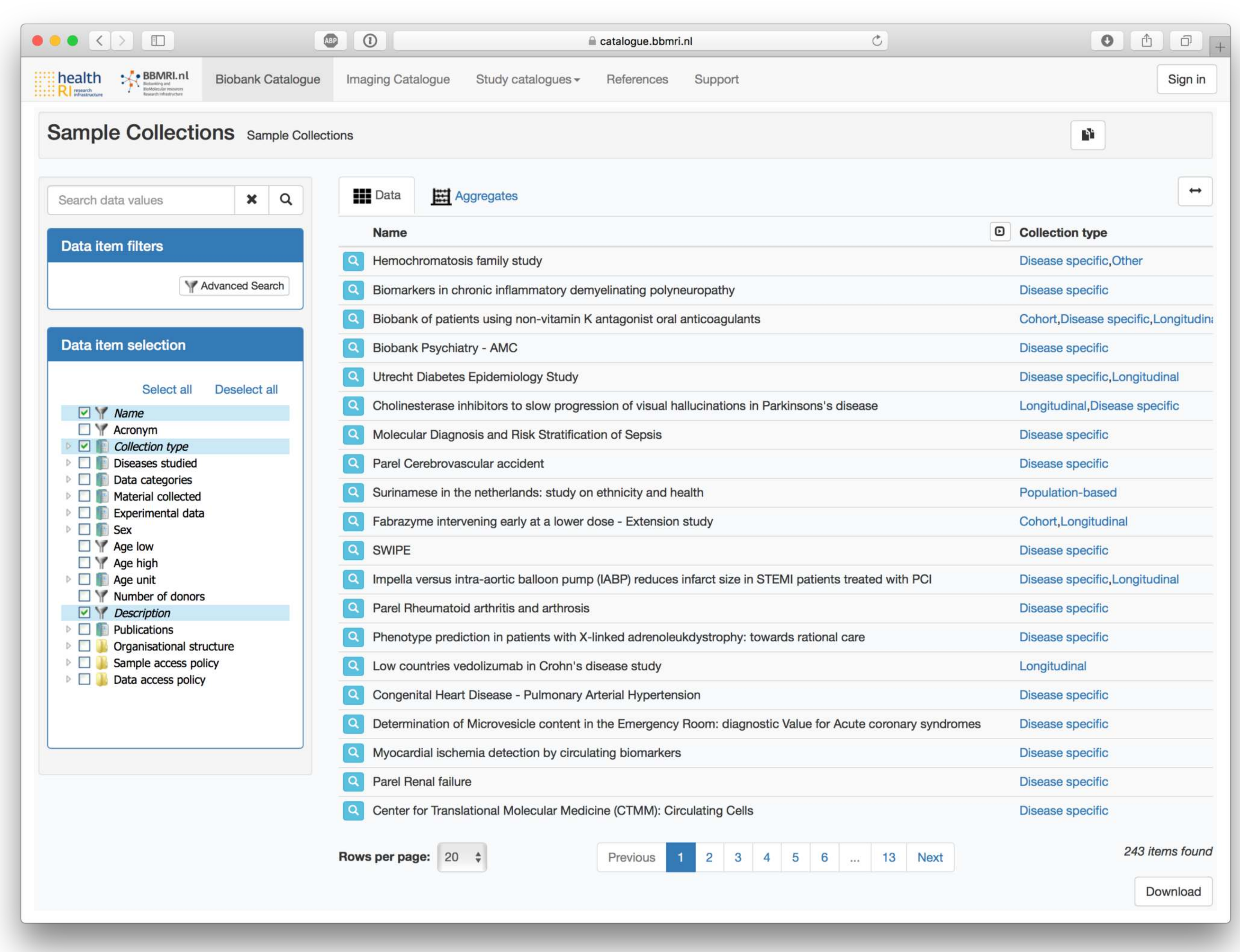

https://catalogue.bbmri.nl/

$\checkmark$ Curated data

$\checkmark$ Link with local catalogues

$\checkmark$ Integration with Podium request workflow

$\checkmark$ Integration with BBMRI-ERIC Directory FAIR Data Point

\section{PODIUM REQUEST WORKFLOW}

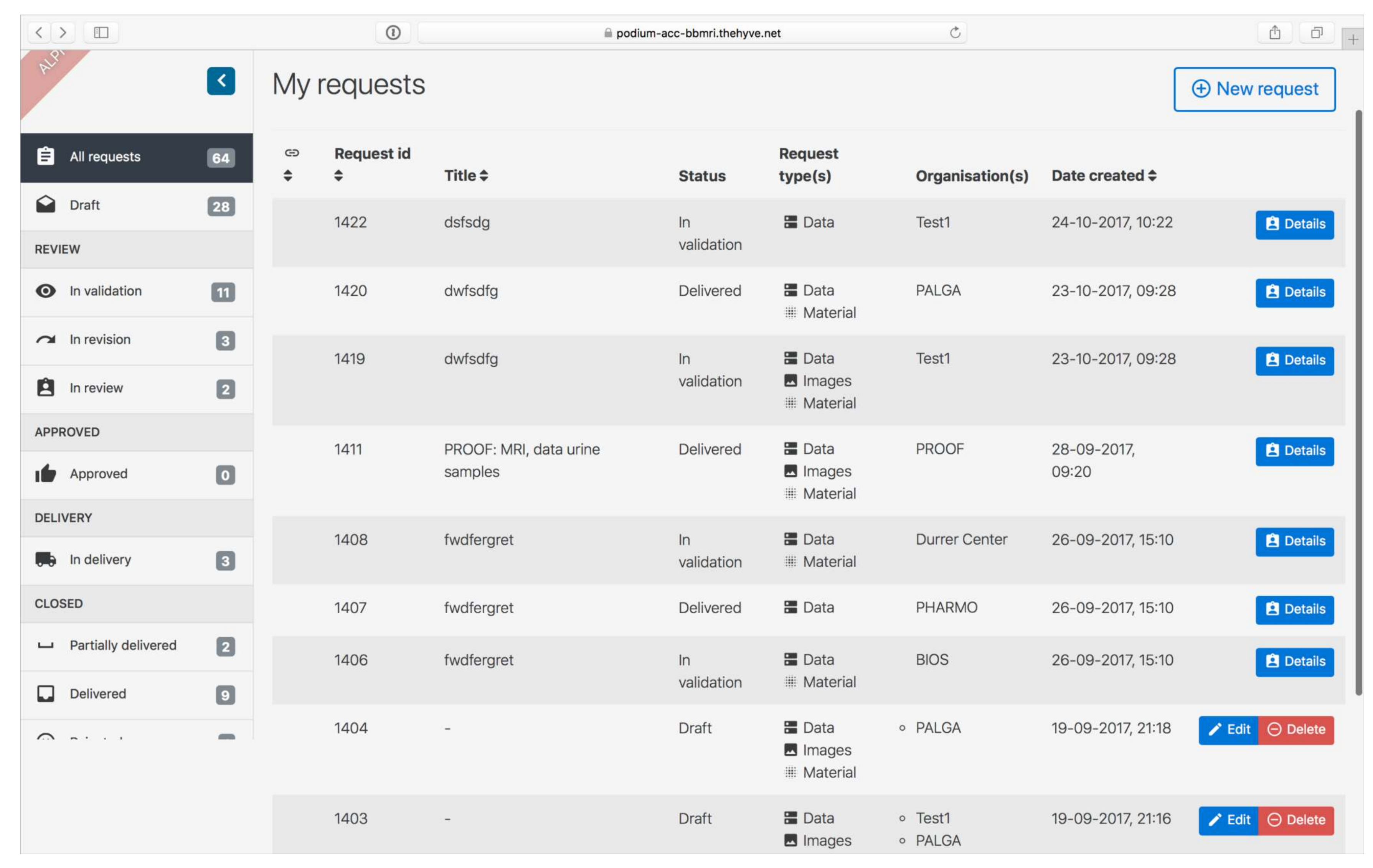

https://podium.bbmri.nl 